\title{
REVIEW
}

\section{Neutrino oscillation studies with reactors}

\author{
P. Vogel ${ }^{1}$, L.J. Wen ${ }^{2} \&$ C. Zhang ${ }^{3}$
}

Nuclear reactors are one of the most intense, pure, controllable, cost-effective and wellunderstood sources of neutrinos. Reactors have played a major role in the study of neutrino oscillations, a phenomenon that indicates that neutrinos have mass and that neutrino flavours are quantum mechanical mixtures. Over the past several decades, reactors were used in the discovery of neutrinos, were crucial in solving the solar neutrino puzzle, and allowed the determination of the smallest mixing angle $\theta_{13}$. In the near future, reactors will help to determine the neutrino mass hierarchy and to solve the puzzling issue of sterile neutrinos.

$\mathrm{N}$ eutrinos, the products of radioactive decay among other things, are somewhat enigmatic, since they can travel enormous distances through matter without interacting even once. Understanding their properties in detail is fundamentally important. Notwithstanding that they are so very difficult to observe, great progress in this field has been achieved in recent decades. The study of neutrinos is opening a path for the generalization of the so-called Standard Model that explains most of what we know about elementary particles and their interactions, but in the view of most physicists is incomplete.

The Standard Model of electroweak interactions, developed in late 1960s, incorporates neutrinos $\left(v_{e}, v_{\mu}, v_{\tau}\right)$ as left-handed partners of the three families of charged leptons $\left(e^{-}, \mu^{-}\right.$, $\left.\tau^{-}\right)$. Since weak interactions are the only way neutrinos interact with anything, the un-needed right-handed components of the neutrino field are absent in the Model by definition and neutrinos are assumed to be massless, with the individual lepton number (that is, the number of leptons of a given flavour or family) being strictly conserved. This assignment was supported by the lack of observation of decays like $\mu^{+} \rightarrow e^{+}+\gamma$ or $K_{L} \rightarrow e^{ \pm}+\mu^{\mp}$, despite the long search for them.

The discovery of neutrino oscillations over the past several decades proved that these assumptions were incorrect. That discovery represents one of the very few instances that show that the Standard Model is indeed incomplete. The phenomenon of neutrino oscillations means that neutrinos have a finite mass, albeit very small, and that lepton flavour is not a conserved quantity. Box 1 explains the basic physics of neutrino oscillations and their relation with neutrino masses, and introduces the parameters used in the oscillation formalism. Determination of all their values, with ever increasing accuracy, was and continues to be the main goal of neutrino experiments. The current experimental values of the mass-squared differences $\Delta m_{i j}^{2}$ and the mixing angles $\theta_{i j}$ can be found in the latest editions of the Review of Particle Physics ${ }^{1}$ and is also shown in Box 1 . Historically, the concept of neutrino oscillations was first considered by Pontecorvo ${ }^{2,3}$ and by Maki, Nakagawa and Sakata ${ }^{4}$, hence the neutrino mixing matrix is usually called the PMNS matrix.

The study of reactor neutrinos played a very significant part in the discovery and detailed study of neutrino oscillations and will continue to be essential to its further progress. Here we

\footnotetext{
${ }^{1}$ Kellogg Radiation Laboratory, California Institute of Technology, Pasadena, California 91125, USA. ${ }^{2}$ Institute of High Energy Physics, Beijing 100049, China.

3 Brookhaven National Laboratory, Upton, New York 11973, USA. Correspondence should be addressed to C.Z. (email: chao@bnl.gov)
} 
briefly review the main points of this saga. Figure 1 illustrates how the flavour composition of the reactor neutrino flux, starting as pure $\bar{v}_{e}$ at production (see the next section for details), is expected

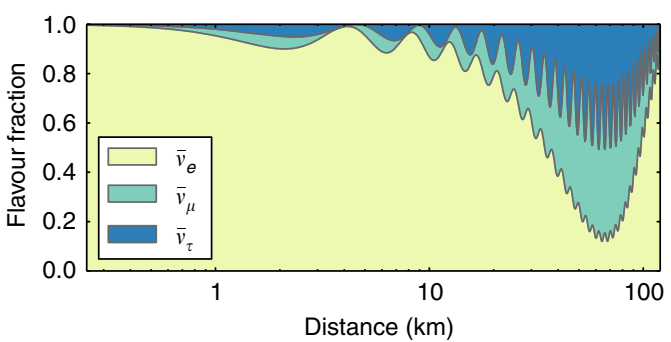

Figure 1 | Illustration of neutrino oscillations. The expected flavour composition of the reactor neutrino flux, for neutrinos of $4 \mathrm{MeV}$ energy used as an example, is plotted as a function of distance to the reactor cores. The fraction of neutrino flavours is calculated on the basis of the neutrino oscillation theory introduced in Box 1. Reactor neutrino oscillation experiments are placed at different baselines to measure the oscillation features driven by different mechanisms. The experiments are usually categorized as follows: very short-baseline $(L \sim 10 \mathrm{~m})$; short-baseline $(L \sim 100 \mathrm{~m})$; kilometre-baseline $(L \sim 1 \mathrm{~km})$; medium-baseline $(L \sim 50 \mathrm{~km})$; and long-baseline $(L>100 \mathrm{~km})$ experiments. Details of the past and future reactor experiments, their goals and achievements are the main topics of this Review. to oscillate as a function of distance. Experimental verification of this behaviour, and quantitative analysis of the results, are the main topics discussed below.

The existence of neutrinos was predicted by Pauli already in 1930 (ref. 5) in his famous letter attempting to explain the continuous electron energy distribution in nuclear beta decay. However, it was not until 1953-1959 that Reines and Cowan ${ }^{6-8}$ were able to show that neutrinos were real particles. Their observation used the electron antineutrinos emitted by a nuclear reactor and started a long tradition of fundamental discoveries using reactor $\bar{v}_{e}$ 's.

In the early experiments, detectors were placed at distances $L \leq 100 \mathrm{~m}$ (refs 9-15; for a review see ref. 16). These pioneering short-baseline experiments, in agreement with the later established three-neutrino oscillation theory, did not observe variations with distance; however, they were important for the understanding of the reactor neutrino flux and spectrum. The KamLAND experiment ${ }^{17-19}$ in the 2000 s convincingly showed that the earlier solar neutrino measurements were indeed caused by oscillations. It demonstrated for the first time that the reactor neutrinos indeed oscillate, that is, that the $\bar{v}_{e}$ component changes with $L / E_{v}$, as explained in Box 1 . It also allowed the most accurate determination of the mass-squared difference $\Delta m_{21}^{2}$.

In the next generation of reactor experiments including Daya Bay $^{20,21}, \mathrm{RENO}^{22}$ and Double Chooz ${ }^{23,24}$, the longstanding

\section{Box 1 | Neutrino oscillations.}

Neutrinos are produced with a definite 'flavour': $v_{e}, v_{\mu}$ or $v_{\tau}$. For example, in nuclear $\beta$-decay electron antineutrinos $\left(\bar{v}_{e}\right)$ are always produced together with an electron. Similarly, if a positively charged muon $\left(\mu^{+}\right)$is produced in the decay of the meson $\pi^{+}$, the muon neutrino $\left(v_{\mu}\right)$ is always produced as well. However, if neutrinos have a finite mass, the flavour composition of a neutrino beam could vary regularly as a function of the distance and energy. This behaviour, called neutrino oscillation, is a subtle consequence of quantum mechanics that postulates that a neutrino of a given flavour need not be a state of a definite mass, but instead could be a coherent superposition of several states of definite masses. Here we explain the basic ideas of this phenomenon.

Let us assume that there are only two massive neutrinos $v_{i}, i=1,2$ with different masses $m_{i}$. In quantum mechanics the development of a state $\psi$ with momentum $p$ is governed by $\psi(t)=\psi(0) e^{i p L}$. (We use the usual system of units where $\hbar=c=1$ ). For highly relativistic neutrinos $p=\sqrt{E^{2}-m^{2}} \sim E-m^{2} / 2 E$. Thus, when neutrinos propagate in vacuum over a distance $L$, each acquires the $\operatorname{phase} v_{i}(L)=v_{i}(0) \exp \left(-i m_{i}^{2} L / 2 E\right)$. (The overall phase is skipped. Additional phases are acquired when neutrinos propagate in matter, the so-called 'MSW effect' ${ }^{\prime 28,129}$, which for simplicity will not be discussed here.) Assume further that the flavour neutrinos $v_{e}$ and $v_{\alpha}$ that is, the neutrinos that are the partners of charged leptons in the weak interactions, are coherent superpositions of the states $v_{i}$, that is, $v_{e}=\cos \theta v_{1}+\sin \theta v_{2}$, and analogous but orthogonal combination represents the other flavour neutrino $v_{\alpha}=-\sin \theta v_{1}+\cos \theta v_{2}$. These mixtures are characterized by the parameter $\theta$, the so-called mixing angle.

Consider now a beam of neutrinos that at $L=0$ is pure $v_{e}$. Then

$$
v_{e}(L)=\cos \theta e^{-i m_{1}^{2} L / 2 E} v_{1}(0)+\sin \theta e^{-i m_{2}^{2} L / 2 E} v_{2}(0) .
$$

In order to observe this beam at $L$ we need to use the weak interactions. We must therefore project the $v_{i}$ back to the flavour basis $v_{e}$ and $v_{\alpha}$. Thus,

$$
v_{e}(L)=\left[\cos ^{2} \theta e^{-i m_{1}^{2} L / 2 E}+\sin ^{2} \theta e^{-i m_{2}^{2} L / 2 E}\right] v_{e}(0)-\sin \theta \cos \theta\left[e^{-i m_{1}^{2} L / 2 E}-e^{-i m_{2}^{2} L / 2 E}\right] v_{\alpha}(0) .
$$

The probability that we detect $v_{e}$ at the distance $L$ is just the square of the corresponding $v_{e}(0)$ amplitude. The probability of detecting $v_{\alpha}$ is the square of the $v_{\alpha}(0)$ amplitude. After some simple algebra this becomes:

$$
P\left(v_{e} \rightarrow v_{e}\right)=1-\sin ^{2} 2 \theta \sin ^{2} \frac{\Delta m^{2} L}{4 E}, P\left(v_{e} \rightarrow v_{\alpha}\right)=\sin ^{2} 2 \theta \sin ^{2} \frac{\Delta m^{2} L}{4 E},
$$

where $\Delta m^{2}=m_{2}^{2}-m_{1}^{2}$ is the difference of the squares of the neutrino masses.

We see that, provided $\Delta m^{2} \neq 0$ and $\theta \neq 0$ or $\pi / 2$, the composition of the neutrino beam oscillates as a function of $L / E_{v}$ with the amplitude $\sin ^{2} 2 \theta$ and wavelength

$$
L_{\mathrm{osc}}=4 \pi \frac{E}{\Delta m^{2}} \equiv L_{\mathrm{osc}}(\mathrm{m})=\frac{2.48 E(\mathrm{MeV})}{\Delta m^{2}\left(\mathrm{eV}^{2}\right)} .
$$

Observation of neutrino oscillations, consequently, constitutes a proof that at least some of the neutrinos have a finite mass and that the superposition is a nontrivial one. Generalization to the realistic case of three neutrino flavours and three states of definite mass is straightforward. The corresponding mixing is then characterized by three mixing angles $\theta_{12}, \theta_{13}$ and $\theta_{23}$, one possible CP violating phase $\delta_{\mathrm{CP}}$ and two mass square differences $\Delta m_{21}^{2}$ and $\Delta m_{32}^{2}$. As of 2014, in the latest edition of the Review of Particle Physics ${ }^{1}$, the best measured values using existing data are

$$
\begin{aligned}
\sin ^{2}\left(2 \theta_{12}\right) & =0.846 \pm 0.021, \sin ^{2}\left(2 \theta_{13}\right)=0.093 \pm 0.008, \sin ^{2}\left(2 \theta_{23}\right)=0.999_{-0.018}^{+0.001}, \\
\Delta m_{21}^{2} & =(7.53 \pm 0.18) \times 10^{-5} \mathrm{eV}^{2}, \text { and } \Delta m_{32}^{2}=(2.44 \pm 0.06) \times 10^{-3} \mathrm{eV}^{2}
\end{aligned}
$$

(assuming $m_{3}>m_{2}$ ). The mass ordering between $m_{3}$ and $m_{2}$, often referred to as the neutrino mass hierarchy, and the value of $\delta_{\mathrm{CP}}$, are currently still unknown.

Neutrino oscillations have no classical analogue. They are purely quantum-mechanical phenomena, the consequence of the coherence of the superposition of states. 
puzzle of the value of the mixing angle $\theta_{13}$ was successfully resolved; it turns out that its value $\theta_{13} \sim 8.9^{\circ}$ (or $\sin ^{2} 2 \theta_{13}=0.093$ (ref. 1)) is not as small as many physicists expected. That discovery opened opportunities for further experiments that should eventually let us determine the so-far missing fundamental features of the oscillations, the neutrino mass hierarchy $(\mathrm{MH})$ and the phase $\delta_{\mathrm{CP}}$ that characterizes the possible CP (charge and parity) violation. The planned reactor experiments, JUNO ${ }^{25}$ and RENO-50 (ref. 26), promise to be an important step on this path.

Most of the oscillation results are well described by the simple three-neutrino generation hypothesis. However, there are a few anomalous indications, the so-called reactor antineutrino anomaly ${ }^{27}$ among them, that cannot be explained this way. If confirmed, they would indicate the existence of additional fourth, or fifth, and so on neutrino families called sterile neutrinos. These neutrinos lack weak interactions and would be observable only when mixing with the familiar active neutrinos. The proposed very short-baseline reactor experiments at distances $\sim 10 \mathrm{~m}$ will test whether this fascinating possibility is realistic or not.

The discovery of neutrino oscillations is one of the most important events in the field of particle physics at the present time. In this work we briefly review the contribution to this achievement of the experiments involving neutrinos emitted by nuclear reactors. First, we show how the reactor flux and its spectrum are determined. Then, we describe the success of the KamLAND experiment that is complementary to the exploration of solar neutrino oscillations, and the determination of the smallest mixing angle $\theta_{13}$ using three independent reactor experiments.

While those achievements involved considerable effort, answering the remaining open questions is even more complicated. We begin by describing the planned large reactor experiments, JUNO and RENO-50, aiming at the difficult determination of the so far unknown neutrino $\mathrm{MH}$ or mass ordering. We then touch on the future very short-baseline reactor experiments aiming to test the tentative and unexpected possibility that additional light-sterile neutrinos might exist.

\section{Reactor neutrino flux and spectrum}

Nuclear reactors derive their power from fission. The fission fragments are neutron-rich and undergo a cascade of $\beta$ decays. Each fission is accompanied by approximately six $\beta$ decays, producing an electron and electron antineutrino each. The decay energy, typical for the nuclear $\beta$ decay, is a few $\mathrm{MeV}$, rarely exceeding $\sim 8 \mathrm{MeV}$. Since a typical power reactor core has thermal power of about $3 \mathrm{GW}_{\text {th }}$, and produces $\sim 200 \mathrm{MeV}$ of energy in each fission, the typical yield of $\bar{v}_{e}$ at equilibrium is $\sim 6 \times 10^{20} \bar{v}_{e}$ core $^{-1} \mathrm{~s}^{-1}$. Reactors are therefore powerful sources of low-energy $\bar{v}_{e}$ 's.

Neutrinos can be detected through charged current interactions when they produce charged particles, electron (mass $0.511 \mathrm{MeV}$ ), muon (mass $105.7 \mathrm{MeV}$ ) or tau (mass $1776.8 \mathrm{MeV}$ ), with neutrino energy sufficient to produce them. The reactor $\bar{v}_{e}$ energy is low; thus, only reactions producing positrons are possible. Hence, to study neutrino oscillations with nuclear reactors, one must use the disappearance type of tests, that is, measure the flux as a function of the distance $L$ and energy $E_{v}$ (see Box 1 for the detailed formalism) and look for the deviation from the simple geometrical scaling. Traditionally, such measurements were compared with the expected $\bar{v}_{e}$ spectrum of the reactor. Good knowledge of that spectrum, its normalization and the associated uncertainties is essential in that case. To reduce the dependence on the knowledge of the reactor spectrum, more recent experiments $\mathrm{s}^{20,22}$ use two essentially similar detectors, one nearer the reactor and another farther away.
There are two principal and complementary ways to evaluate antineutrino spectra associated with fission. The summation method uses known cumulative fission yields $Y_{n}(Z, A, t)$ and combines them with the experimentally known (or theoretically deduced) branching ratios $b_{n, i}\left(E_{0}^{i}\right)$ of all $\beta$-decay branches with end points $E_{0}^{i}$ and a normalized shape function of each of these many thousands of $\beta$ decays, $P_{\bar{v}}\left(E_{\bar{v}}, E_{0}^{i}, Z\right)$,

$$
\frac{d N}{d E_{\bar{v}}}=\sum_{n} Y_{n}(Z, A, t) \sum_{i} b_{n, i}\left(E_{0}^{i}\right) P_{\bar{v}}\left(E_{\bar{v}}, E_{0}^{i}, Z\right) .
$$

There are several difficulties with this method. The branching ratios and end point energies are sometimes poorly known at best, in particular for the short-lived fragments with large $Q$ values and many branches. The individual spectrum shape functions $P_{\bar{v}}\left(E_{\bar{v}}, E_{0}^{i}, Z\right)$ require description of the Coulomb distortions including the nuclear finite size effects, weak magnetism and radiative corrections. In addition, not all decays are of the allowed type. There are numerous ( 25\%) first forbidden decays involving parity change, where the individual spectrum shapes are much more difficult to evaluate.

The other method uses the experimentally determined spectrum of electrons associated with fission of the principal reactor fuels. That spectrum has been measured at ILL Grenoble for the thermal neutron fission of ${ }^{235} \mathrm{U},{ }^{239} \mathrm{Pu}$ and ${ }^{242} \mathrm{Pu}$ (refs 28-30) and recently also for the fast neutron fission of ${ }^{238} \mathrm{U}$ in Munich ${ }^{31}$. These electron spectra are then transformed into the $\bar{v}_{e}$ spectra using the obvious fact that these two leptons share the total energy of each $\beta$-decay branch. The transformation is on the basis of fitting first the electron spectra to a set of 30 or more virtual branches, with the equidistant end point spacing, determining from the fit their branching ratios. The conversion to the $\bar{v}_{e}$ spectrum is performed in each of these virtual branches. That conversion is based on the assumption that the electron spectrum is known precisely. When all virtual branches are put together, one has to also take into account that different nuclear charges $Z$ contribute with different weights to different electron and $\bar{v}_{e}$ energies. While the conversion would introduce only minimum uncertainty if all decays would be of the allowed shape, the presence of the first forbidden decays introduces additional uncertainty whose magnitude is difficult to determine accurately.

The summation method was used initially in refs $32-36$ and in the more recent version in ref. 37. The conversion method was first used in refs 28-30, more details can be found in ref. 38 and the more recent version in ref. 39. Naturally, the thermal power of the reactor and its time-changing fuel composition must be known, as must the energy associated with fissions of the isotopes ${ }^{235} \mathrm{U},{ }^{239} \mathrm{Pu},{ }^{241} \mathrm{Pu}$ and ${ }^{238} \mathrm{U}$. In addition, as already mentioned, small corrections to the spectrum shape of individual $\beta$-decay branches due to the radiative correction, weak magnetism, nuclear size, and so on must be correctly included. Difficult to do accurately, but of a particular importance, is to take into account the spectrum shape of the numerous first forbidden $\beta$-decays ${ }^{40}$. The overall uncertainty in the flux was estimated in refs 37,39 to be $\sim 2 \%$; however, when the first forbidden decays are included it is estimated in ref. 40 that the uncertainty increases to $\sim 5 \%$.

In essentially all reactor neutrino oscillation studies, the $\bar{v}_{e}$ are detected using the inverse neutron $\beta$-decay reaction

$$
\bar{v}_{e}+p \rightarrow e^{+}+n, \sigma=9.53 \frac{E_{e} p_{e}}{\mathrm{MeV}^{2}}(1+\text { corr. }) \times 10^{-44} \mathrm{~cm}^{2},
$$

whose cross-section is accurately known ${ }^{41,42}$ and depends primarily on the known neutron decay half-life. (At the same time the recoil, radiative corrections and so on must also be taken into account.) Since the neutron is so much heavier than the available energy, its kinetic energy is quite small (tens of $\mathrm{keV}$ ) and 


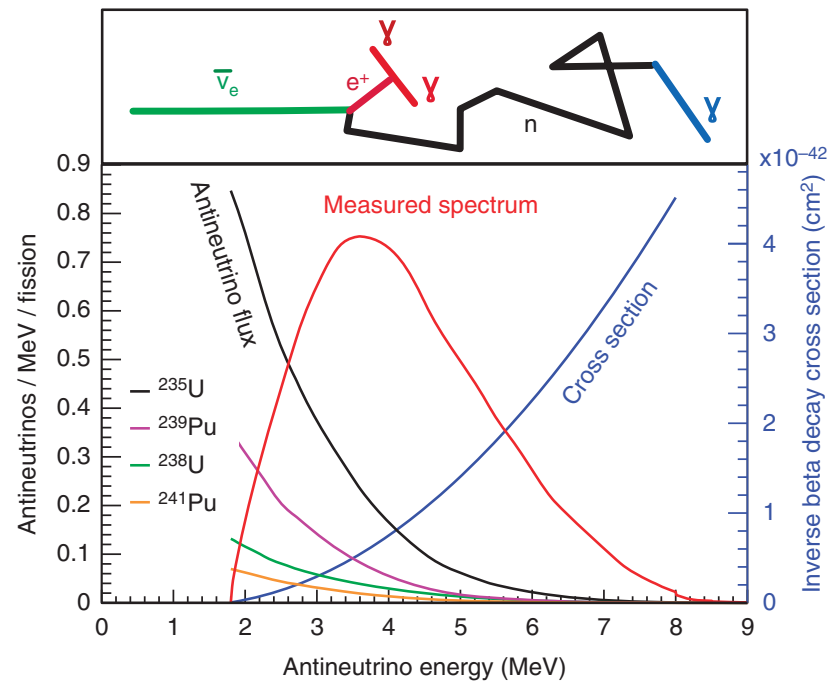

Figure 2 | Detection of reactor $\overline{\boldsymbol{v}}_{\mathrm{e}}$. In the bottom of the figure, the reactor $\bar{v}_{e}$ flux from the individual isotopes ${ }^{37,39}$, weighted by their typical contribution to the total flux in a commercial reactor, is shown. The detection of $\bar{v}_{e}$ relies on the inverse beta decay reaction, whose crosssection ${ }^{41,42}$ is shown as the blue curve. Their product is the interaction spectrum measured by the detectors, shown as the red curve. The steps involved in the detection are schematically drawn in the top of the figure. The $\bar{v}_{e}$ interacts with a proton, becoming a positron $\left(e^{+}\right)$and a neutron. The $e^{+}$quickly deposits its energy and annihilates into two 511-keV $\gamma$-rays, which gives a prompt signal. The neutron scatters in the detector until being thermalized. It is then captured by a proton $\sim 200 \mu$ s later and releases a $2.2-\mathrm{MeV} \gamma$-ray (the capture time can be significantly reduced by the doping of isotopes with very large neutron capture cross-section such as gadolinium). The detection of this prompt-delayed signal pair indicates an $\bar{v}_{e}$ candidate.

thus the principal observables are the number and energy of the positrons. Most importantly, the correlated observation of the positrons and the delayed neutron captures is a powerful tool for background suppression. Note that the reaction (2) has a threshold of $1.8 \mathrm{MeV}$, only $\bar{v}_{e}$ with energy larger than that can produce positrons.

In Fig. 2 we illustrate the energy dependence of the reactor $\bar{v}_{e}$ flux, the detection reaction cross-section and their product, that is, the measured antineutrino spectrum. The contributions of the individual isotopes to the $\bar{v}_{e}$ flux, weighted by their typical contribution to the reactor power are also shown. The top part of the figure schematically indicates the steps involved in the $\bar{v}_{e}$ capture on proton reaction.

\section{Exploring solar neutrino oscillations on earth}

Since the late 1960s, a series of solar neutrino experiments ${ }^{43-47}$, using charged current reactions, have observed a large deficit of the solar $v_{e}$ flux relative to the Standard Solar Model (SSM) ${ }^{48}$ prediction. It appeared that more than half of the solar neutrinos were missing. This was referred to as the 'Solar Neutrino Problem'. In 2001, the SNO solar neutrino experiment ${ }^{49}$, for the first time, successfully measured the total flux of all three neutrino flavours $v_{e}, v_{\mu}$ and $v_{\tau}$ from the Sun through the neutral current channel $v+d \rightarrow v+p+n$ using heavy water as a target, that yielded results consistent with the SSM. The SNO result is considered the 'smoking gun' evidence of the neutrino oscillation explanation to the Solar Neutrino Problem-the solar neutrinos, produced as electron-neutrinos from fusion and other reactions in the central region of the Sun, are transformed into other flavours when they arrive at the Earth.
The solar neutrino experiments allowed several possible solutions in the oscillation parameter space of $\theta_{12}$ and $\Delta m_{21}^{2}$. A precise measurement of these parameters and demonstration of the oscillatory feature, however, was hindered by the relatively large uncertainty of the solar $v_{e}$ flux predicted by the SSM, the large matter effect inside the Sun and the extremely long distance the neutrinos travel. A reactor neutrino experiment, measuring the same disappearance channel as the solar neutrino experiments assuming CPT invariance, overcomes these difficulties. With well-understood and controllable $\sim \mathrm{MeV} \bar{v}_{e}$ source, a reactor experiment at $\sim 100 \mathrm{~km}$ baselines can explore with high precision the so-called 'Large Mixing Angle' parameter region suggested by the solar neutrino experiments. To do that, the KamLAND experiment ${ }^{17}$ was built in early 2000 s to explore the solar neutrino oscillations on Earth.

To shield against cosmic rays, the KamLAND detector was placed at the site of the former Kamiokande experiment ${ }^{46}$ under the summit of Mt. Ikenoyama in the Japanese Alps. The vertical overburden is 2,700 metre water equivalent (m.w.e.). It is surrounded by 55 Japanese nuclear reactor cores, which then produced $\sim 30 \%$ of the total electricity in Japan. The $\bar{v}_{e}$ fluxweighted average baseline is $\sim 180 \mathrm{~km}$, well suited for KamLAND to study the parameters suggested by the solar neutrino experiments. The reactor operation information such as thermal power, fuel burn-up and fuel exchange, and enrichment records, were provided by all Japanese reactors, which allowed KamLAND to calculate the instantaneous fission rate of each isotope accurately.

The KamLAND detector consists of 1 kton of highly purified liquid scintillator (LS), enclosed in a 13-m-diameter transparent balloon suspended by ropes in mineral oil (MO). The MO is housed inside a 18-m-diameter stainless steel (SS) sphere, where an array of 1,325 17-inch and 55420 -inch photomultiplier tubes (PMTs) is mounted. The MO shields the inner LS region from external radiation from PMTs and SS. Purified water ( 3.2 kton) is used to provide further shielding against ambient radiation and it operates as an active cosmic muon veto detector. With regular central-axis deployments of radioactive sources and dedicated offaxis deployments ${ }^{50}$, KamLAND achieved an excellent position resolution of $12 \mathrm{~cm} / \sqrt{E(\mathrm{MeV})}$, energy resolution of $6.5 \% / \sqrt{E(\mathrm{MeV})}$ and absolute energy-scale uncertainty of $1.4 \%$.

Even with such powerful reactor $\bar{v}_{e}$ sources and a massive detector, the long baseline suppresses the expected signal at KamLAND to only about one reactor $\bar{v}_{e}$ event per day. In comparison, the background from internal and external radioactivity is one million times higher. The experiment is only possible owing to the powerful coincidence signature (the positron followed by the delayed neutron capture $\gamma$ ) of inverse beta decay, as illustrated in Fig. 2. A time difference of less than $1 \mathrm{~ms}$ and distance less than $2 \mathrm{~m}$ between the prompt and delayed signals is required in the analysis. Only the innermost 6 -m-radius scintillator region is used to reduce the accidental coincidence from external radioactivity. Information about the event energy, position and time was used to further reduce the accidental background to $\sim 5 \%$ of the candidates.

The other dominant background $(\sim 10 \%)$ at KamLAND is caused by the ${ }^{13} \mathrm{C}(\alpha, n){ }^{16} \mathrm{O}$ reaction where the $\alpha$-decay comes from ${ }^{210} \mathrm{Po}$, a decay product of ${ }^{222} \mathrm{Rn}$ that is naturally present in the air and many materials as traces, but is sufficient to induce a measurable contamination of the scintillator during its production. The neutron scattering off proton or ${ }^{16} \mathrm{O}^{*}$ de-excitation produces a prompt signal, followed by a neutron capture-delayed signal. This then mimics a true $\bar{v}_{e}$ event. The rest of the backgrounds include the following: the antineutrinos produced in the decay chains of ${ }^{232} \mathrm{Th}$ and ${ }^{238} \mathrm{U}$ in the Earth's mantle and crust, so-called geoneutrinos; the cosmogenic beta-delayed 


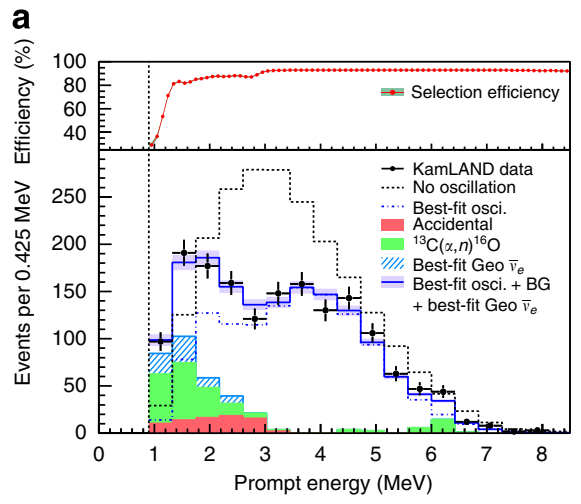

b

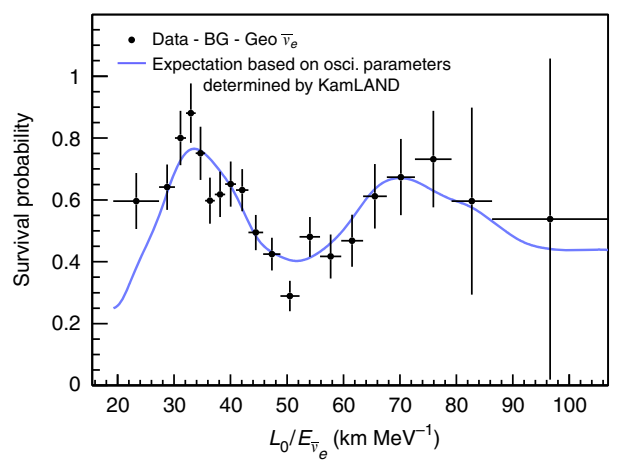

Figure 3 | Results from KamLAND. (a) The data points show the measured prompt energy spectrum of $\bar{v}_{e}$ candidate events. The shaded histograms show the expected backgrounds. The expected reactor spectra without oscillation and with best-fit oscillation are shown as the dashed histogram. All histograms incorporate the energy-dependent selection efficiency, which is shown on the top. Only $\sim 60 \%$ of reactor $\bar{v}_{e}$ 's are observed relative to the no-oscillation expectation. (b) The data points show the ratio of the background-subtracted $\bar{v}_{e}$ spectrum to the expectation for no-oscillation as a function of $L_{0} / E_{v}$. $L_{0}$ is the effective baseline taken as a flux-weighted average $\left(L_{0}=180 \mathrm{~km}\right)$. The spectrum indicates almost two cycles of periodic feature as expected from neutrino oscillations. The oscillation survival probability using the best estimates of $\theta_{12}$ and $\left|\Delta m_{21}^{2}\right|$ is given by the blue curve. The curve deviates from the perfect sinusoidal $L / E$ dependence since KamLAND has multiple baselines. Panels $\mathbf{a}, \mathbf{b}$ are reproduced, with permission, from ref. 19 . Copyright 2008 by the American Physical Society.

neutron emitters ${ }^{9} \mathrm{Li}$ and ${ }^{8} \mathrm{He}$; the fast neutrons from muons passing through the surrounding rock; as well as atmospheric neutrinos.

Figure 3a shows the prompt energy spectrum of $\bar{v}_{e}$ candidate events, observed with $2.9 \mathrm{kton}$ - year exposure, overlaid with the expected reactor $\bar{v}_{e}$ and background spectra. A total of 1,609 events were observed, which is only $\sim 60 \%$ of the expected signal if there are no oscillations. The ratio of the backgroundsubtracted $\bar{v}_{e}$ candidate events to no-oscillation expectation is plotted in Fig. $3 \mathrm{~b}$ as a function of $L / E_{v}$. The spectrum indicates almost two cycles of the periodic feature expected from neutrino oscillations, strongly disfavouring other explanations of the $\bar{v}_{e}$ disappearance.

The KamLAND results ${ }^{17-19}$ are highly consistent with the solar neutrino experiments, and have pinned down the solar neutrino oscillation solution to the Large Mixing Angle region. When combined with the results from SNO, they yield the most precise measurements of $\tan ^{2} \theta_{12}=0.47_{-0.05}^{+0.06}$ and $\Delta m_{21}^{2}=7.59_{-0.21}^{+0.21} \times 10^{-5} \mathrm{eV}^{2}$. This is a great example of the complementarity between different types of experiments. The SNO and KamLAND's first results came out within $\sim 18$ months of each other, with the solar experiment being more sensitive to the mixing angle $\theta_{12}$ and the reactor experiment to the masssquared difference $\Delta m_{21}^{2}$. The observation of the same effect with two different sources on such different scales provides compelling evidence for neutrino oscillations.

\section{Searching for the smallest oscillation angle}

In contrast to the Cabibbo-Kobayashi-Maskawa matrix in quark mixing, where all three mixing angles are very small ${ }^{1}$, the mixing angles in the neutrino-mixing matrix appear to be large: $\theta_{232}$, measured by the atmospheric ${ }^{11}$ and long-baseline accelerator ${ }^{23}$ neutrino experiments, is consistent with $45^{\circ}$, which corresponds to maximal mixing; and $\theta_{12}$, measured by the solar neutrino experiments and KamLAND, is $\sim 33^{\circ}$. It was therefore natural to expect that the third mixing angle, $\theta_{13}$, might be of similar magnitude.

The cleanest way to measure $\theta_{13}$ is through kilometre-baseline reactor neutrino oscillation experiments. A non-zero $\theta_{13}$ will cause a deficit of the $\bar{v}_{e}$ flux at $\sim 1-2 \mathrm{~km}$ baseline, as indicated in Fig. 1 . The size of the deficit is directly proportional to the value of $\sin ^{2} 2 \theta_{13}$. Unlike accelerator neutrino experiments, the reactor measurements are independent of the CP phase and $\theta_{23}$, and only slightly dependent on the neutrino $\mathrm{MH}$ and matter effect. A high precision measurement can therefore be achieved.

In the 1990s, two first-generation kilometre-baseline reactor experiments, $\mathrm{CHOOZ}^{53}$ and PALO VERDE ${ }^{54}$, were constructed to measure $\theta_{13}$. The $\mathrm{CHOOZ}$ detector was built at a distance of $\sim 1,050 \mathrm{~m}$ from the two reactors of the $\mathrm{CHOOZ}$ power plant of Électricité de France in the Ardennes region of France. It took data from April 1997 to July 1998. The PALO VERDE detector was built at distances of 750,890 and $890 \mathrm{~m}$ from the three reactors of the Palo Verde Nuclear Generating Station in the Arizona desert of the United States. It took data between October 1998 and July 2000. Surprisingly, neither experiment was able to observe the $\bar{v}_{e}$ deficit caused by $\theta_{13}$ oscillation. As a result, only an upper limit of $\sin ^{2} 2 \theta_{13}<0.10$ at $90 \%$ C.L. was obtained ${ }^{53}$.

The null results from $\mathrm{CHOOZ}$ and PALO VERDE, combined with the measured values of $\theta_{23}$ and $\theta_{12}$, motivated many phenomenological speculations of neutrino-mixing patterns such as bimaximal and tribimaximal mixing ${ }^{55,56}$. In most of these theories, $\theta_{13}$ is either zero or very small. A direct consequence of a vanishing $\theta_{13}$ is that the $\mathrm{CP}$ violation in the leptonic sector, even if large, can never be observed in the neutrino oscillation experiments. The importance of knowing the precise value of $\theta_{13}$ provoked a series of worldwide second-generation kilometrebaseline reactor experiments in the twenty-first century, including Double Chooz ${ }^{23}$ in France, RENO ${ }^{22}$ in Korea and Daya Bay ${ }^{20}$ in China, to push the sensitivity to $\theta_{13}$ considerably below $10^{\circ}$. Table 1 summarizes some of the key parameters of the five aforementioned experiments.

A common technology used in both the first- and secondgeneration experiments is the gadolinium-loaded liquid scintillator as the $\bar{v}_{e}$ detection target. Gd has a high thermal neutron capture cross-section. With $\sim 0.1 \%$ gadolinium loading, the neutron capture time is reduced to $\sim 28$ from $\sim 200 \mu$ s for the unloaded scintillator (as used in KamLAND). Furthermore, Gd de-excitation after the neutron capture releases an $8-\mathrm{MeV}$ gamma-ray cascade, which gives a delayed signal well above natural radioactivity (in contrast, neutron capture on a proton releases a single $2.2-\mathrm{MeV} \gamma$ ). The accidental coincidence background is therefore drastically reduced. 
Addition of near detectors at baselines of a few hundred metres is the most significant improvement of the second-generation experiments over the previous ones. As discussed above, the uncertainty in predicting the reactor antineutrino flux is relatively large $(2-5 \%)$. This flux uncertainty, however, can be largely eliminated by the relative measurement between near and far detectors. The Double Chooz experiment expands $\mathrm{CHOOZ}$ by adding a near detector at a distance of $\sim 400 \mathrm{~m}$. The installation of that near detector, however, was delayed because of civil construction. Double Chooz started taking data in May 2011 with only a far detector, and used the Bugey 4 measurement ${ }^{14}$ to normalize the reactor flux. The RENO experiment was built near the six reactors of the Yonggwang nuclear power plant (NPP) in Korea. The two identical detectors were located at 290 and $1,380 \mathrm{~m}$, respectively, from the centre of the reactor array. RENO started taking data in August 2011. The Daya Bay experiment was built near the six reactors of the Daya Bay NPP in southern China. Daya Bay had eight identical antineutrino detectors (ADs). Two ADs were placed at $\sim 360 \mathrm{~m}$ from the two Daya Bay reactor cores. Two ADs were placed at $\sim 500 \mathrm{~m}$ from the four Ling Ao reactor cores. Moreover, four ADs were placed at a far site $\sim 1,580 \mathrm{~m}$ away from the six-reactor complex. This modular detector design allows Daya Bay to largely remove the correlated detector systematics. Daya Bay started taking data in December 2011.

Compared with the first-generation experiments, the secondgeneration experiments have much larger signal statistics by utilizing higher power reactors and larger detectors. Among them, Daya Bay has the largest reactor power $\left(17.4 \mathrm{GW}_{\mathrm{th}}\right)$ and target mass ( 80 tons at the far site), as shown in Table 1 . The underground sites are much deeper to allow better shielding from cosmogenic background, in particular compared with the case of PALO VERDE. Better chemical recipes of the gadolinium-loaded liquid scintillator also improve the overall detector performance and long-term stability.

The second-generation reactor experiments were a huge success. In 2012, all three experiments, Double Chooz, Daya Bay and RENO, reported clear evidence of $\bar{v}_{e}$ disappearance at $\sim$ kilometre baselines after only a few month's running $20,22,23$. In particular, Daya Bay excluded $\theta_{13}=0$ by 5.2 s.d. with 55 days of data $^{20}$. Figure $4 \mathrm{a}$ shows the result of this discovery. The ratio of the detected to expected no-oscillation $\bar{v}_{e}$ signals at the six detectors located in the three experimental halls is plotted as a function of weighted baseline. The signal rate at the far site shows an obvious $\sim 6 \%$ deficit with respect to the near sites, and fits nicely to the theoretical oscillation curve (in red). The precision of

\section{Table 1 | Key parameters of the reactor $\theta_{13}$ experiments.}

\begin{tabular}{|c|c|c|c|c|}
\hline $\mathrm{CHOOZ}^{53}$ & 8.5 & 1,050 & 5 & 300 \\
\hline PALO VERDE ${ }^{54}$ & 11.6 & $750-890$ & 12 & 32 \\
\hline Double Chooz 23 & 8.5 & 400 & 8 & 120 \\
\hline \multirow[t]{2}{*}{ RENO 22} & 16.8 & 290 & 16 & 120 \\
\hline & & 1,380 & 16 & 450 \\
\hline Daya Bay ${ }^{20}$ & 17.4 & 360 & $2 \times 20$ & 250 \\
\hline
\end{tabular}

m.w.e., metre-water-equivalent

The table summarizes the key parameters of the five past and present reactor $\theta_{13}$ experiments, including the reactor thermal power (in gigawatts), distance to reactors, target mass of the detectors and overburden of the underground site (in m.w.e.).
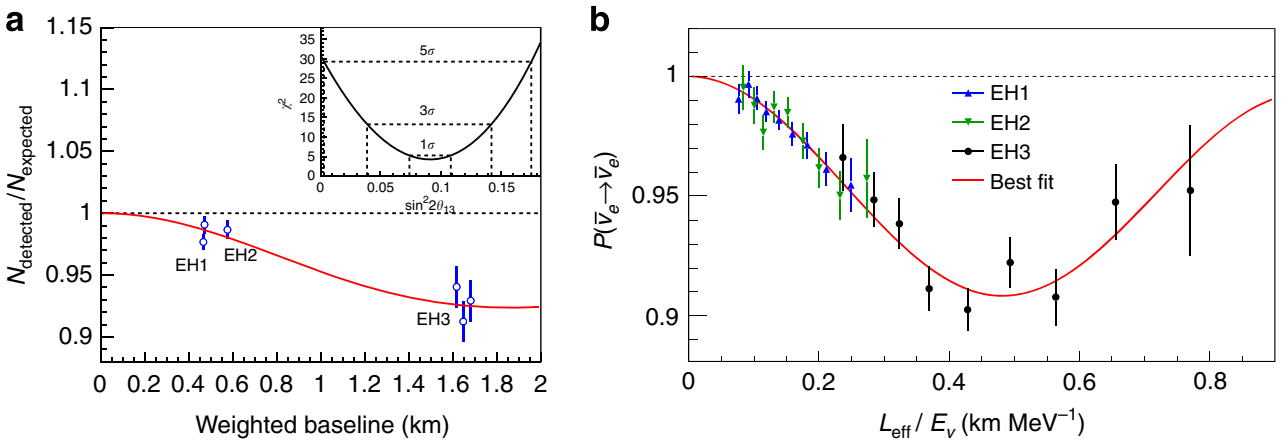

Figure 4 | Results from Daya Bay. (a) The data points show the ratio of the detected to expected no-oscillation $\bar{v}_{e}$ signals at the six antineutrino detectors located in three experimental halls (EHs) as a function of the weighted baseline. An $\sim 6 \%$ signal deficit at the far hall relative to the near halls is observed. The oscillation survival probability at the best-fit value is given by the red curve. The no-oscillation hypothesis $\left(\theta_{13}=0\right)$ is excluded at $5.2 \mathrm{~s}$.d.'s, as shown in the inset. (b) The data points show the ratio of the background-subtracted $\bar{v}_{e}$ spectrum to the expectation for no-oscillation in the three experimental halls as a function of $L_{\text {eff }} / E_{v}$. The effective baseline $L_{\text {eff }}$ is determined for each $\mathrm{EH}$ equating the multicore oscillated flux to an effective oscillated flux from a single baseline. A near-complete cycle of the expected periodic oscillation feature is observed. The oscillation survival probability using the best estimates of $\theta_{13}$ and $\left|\Delta m_{31}^{2}\right|$ is given by the red curve. Panel $\mathbf{a}$ is reproduced, with permission from ref. 20. Copyright 2012 by the American Physical Society. Panel $\mathbf{b}$ is reproduced, with permission from ref. 21. Copyright 2014 by the American Physical Society. 
the $\theta_{13}$ measurement improved quickly with more data. With the data collected in Daya Bay through November 2013 (ref. 57), the best-fit value is $\sin ^{2} 2 \theta_{13}=0.084 \pm 0.005$. Although the last known, the precision in $\theta_{13}$ measurement $(6 \%)$ is now the best among all three mixing angles.

Similar to KamLAND, the ratio of the detected $\bar{v}_{e}$ events to no-oscillation expectation at Daya Bay is plotted in Fig. $4 \mathrm{~b}$ as a function of $L / E_{v}$. The combined data from the three experimental halls show a near-complete cycle of the expected periodic oscillation feature. The smaller amplitude and shorter wavelength of the oscillation, compared with the case of KamLAND, indicate the different oscillation component driven by $\theta_{13}$ and $\Delta m_{31}^{2}$. The best-fit frequency of the oscillation yields $\left|\Delta m_{32}^{2}\right|=$ $2.39_{-0.10}^{+0.11} \times 10^{-3} \mathrm{eV}^{2}$ (assuming normal $\mathrm{MH}$ ), which is consistent and of comparable precision with the measurements of accelerator $v_{\mu}$ and $\bar{v}_{\mu}$ disappearance ${ }^{58,59}$. By the end of 2017, Daya Bay expects to measure both $\sin ^{2} 2 \theta_{13}$ and $\left|\Delta m_{32}^{2}\right|$ to precisions below 3\% (ref. 57).

The discovery of $\theta_{13}$ represents another good example of the complementarity between different types of experiments. The first results from the reactor experiments $20,22,23$ and the accelerator experiments ${ }^{60,61}$ were released within $\sim 9$ months of each other, with the reactor experiments measuring $\bar{v}_{e}$ disappearance and the accelerator experiments measuring $v_{e}$ appearance. Seeing the same $\theta_{13}$-driven effects with different sources of neutrinos at very different energy and baselines is a strong proof of neutrino oscillations.

The longstanding puzzle of the value of $\theta_{13}$ is now successfully resolved. The relatively large value of $\theta_{13}$ opens the gateway for future experiments to determine the neutrino $\mathrm{MH}$ and to measure the CP-violating phase in the leptonic sector.

\section{Determination of neutrino $\mathrm{MH}$}

At present only the absolute values of the neutrino mass-squared differences $\Delta m_{32}^{2}$ and $\Delta m_{31}^{2}$ are known, not their sign. Depending on whether both $\Delta m_{31}^{2}$ and $\Delta m_{32}^{2}$ are positive or whether they are both negative, the neutrino mass ordering is usually referred to as normal or inverted $\mathrm{MH}$, respectively. The neutrino $\mathrm{MH}$ is a problem of fundamental importance ${ }^{62}$ that represents an important step in the formulation of the Generalized Standard Model of particle physics. Its determination will reduce the uncertainty in experiments aiming at the measurement of the CP-violating phase, and it will help in defining the goals of the forthcoming neutrinoless double beta-decay experiments. It will also improve our understanding of core-collapse supernovae.

The reactor $\bar{v}_{e}$ oscillations are modulated by terms that depend on $\Delta m_{31}^{2}$ and $\Delta m_{32}^{2}$. At a medium baseline of $\sim 60 \mathrm{~km}$, multiple small-amplitude, proportional to the $\sin ^{2} 2 \theta_{13}$, oscillation peaks show up on top of the long-wavelength oscillation with the much larger amplitude proportional to the $\sin ^{2} 2 \theta_{12}$, as shown in Fig. 1 . Depending on whether the $\mathrm{MH}$ is normal or inverted, the smallamplitude oscillation pattern shifts slightly. The $\mathrm{MH}$ information can be extracted from this pattern by using a likelihood analysis ${ }^{63}$ or the Fourier transform method ${ }^{64,65}$. Additional information regarding the neutrino $\mathrm{MH}$ could be obtained by combining the reactor oscillation analysis with the long-baseline muon neutrino disappearance one ${ }^{66}$, as the effective mass-squared differences measured there are different combinations of $\Delta m_{31}^{2}, \Delta m_{32}^{2}$ and other oscillation parameters.

Two medium-baseline reactor experiments, $\mathrm{JUNO}^{25}$ in China and RENO-50 (ref. 26) in Korea, have been proposed aiming at the $\mathrm{MH}$ determination, among other goals. JUNO is currently under construction. The experiment is located in Kaiping city, Guangdong province, in southern China. The JUNO detector will be placed underground with a total vertical overburden of 1,800 m.w.e. JUNO will observe antineutrinos from the Yangjiang NPP and the Taishan NPP at equal baselines of $\sim 53 \mathrm{~km}$, as illustrated in Fig. 5, near the maximal $\theta_{12}$-oscillation baseline. The Yangjiang NPP has six reactor cores of $2.9 \mathrm{GW}_{\text {th }}$ each and the Taishan NPP has planned four cores of $4.6 \mathrm{GW}_{\text {th }}$ each, both are under construction. The difference between the baselines to the two NPPs is controlled to less than $500 \mathrm{~m}$ to prevent significant degradation of the $\mathrm{MH}$ discrimination power ${ }^{63}$. JUNO is expected to start data taking in 2020. The proposed RENO-50 (ref. 26) experiment will be located in the city of Naju, $\sim 47 \mathrm{~km}$ from the Hanbit NPP with six cores of $2.8 \mathrm{GW}_{\text {th }}$ each. The detector will be placed at underground of Mt. Guemseong with an overburden of 900 m.w.e. RENO-50 is expected to begin data taking in 2021.

The medium-baseline reactor experiments need to have massive detectors, $\sim 20$ kilotons, in order to collect sufficient $\bar{v}_{e}$ events in a reasonable timescale (a few years). In the following we will primarily use JUNO as an example to illustrate the significant challenges in building such a large experiment. The preliminary design of JUNO includes a central detector submerged in a water pool with the muon trackers installed on top of the pool. The water pool is equipped with PMTs and acts as an active Cherenkov detector for vetoing muons. It also provides passive shielding against the natural radioactivities from the surrounding rock and air. The top trackers provide complementary measurements of the cosmic muons. The central detector consists of 20 kton of LS, contained either in a spherical acrylic tank supported by stainless steel frames, or a thin transparent balloon contained in a stainless steel vessel. The detector looks similar to the one in SNO or KamLAND, but is 20 times larger. In order to collect enough light, the central detector is viewed by $\sim 18,000$ 20-inch PMTs. The PMTs have implosion containers to mitigate the risk of implosion chain reactions. Taking into account the mechanical clearance, the PMTs provide a near maximal surface coverage of 75-78\%. RENO-50 has a similar detector design with 18-kt LS and 15,000 20-inch PMTs.

An energy resolution better than $3 \% / \sqrt{E(\mathrm{MeV})}$ is essential for medium-baseline reactor experiments to maintain the $\mathrm{MH}$ discrimination ability ${ }^{63}$. To achieve that, besides keeping a maximal photocathode coverage, additional technical improvements are necessary. High quantum-efficiency ( 35\%) PMTs are necessary in order to increase the light-detection efficiency. A new type of 20-inch microchannel plate (MCP) PMTs is being developed for JUNO. The light yield and the optical transparency of the LS also need to be improved. Optimizing the concentration of scintillation fluors, purification of the raw solvent and fluors and online $\mathrm{Al}_{2} \mathrm{O}_{3}$ column filtration have been found effective. LS attenuation length of more than $30 \mathrm{~m}$ is desired.

Calibration of the absolute energy scale is crucial. In particular, three main effects cause nonlinear energy response of a LS detector: scintillator quenching, Cherenkov radiation and possible nonlinear electronics response. If the energy nonlinearity correction has large uncertainties, particular residual nonlinear shapes may fake the oscillation pattern with a wrong $\mathrm{MH}^{67}$. The absolute energy scale uncertainty needs to be controlled within a few tenths of a percent, which is challenging from the experience of KamLAND ${ }^{50}$ and Daya Bay ${ }^{57}$. The requirement demands a comprehensive calibration programme for a large detector such as JUNO or RENO-50.

Background control is demanding, in particular because of relatively shallow depth of the experimental sites of JUNO and RENO-50. The sources of background are similar to those of KamLAND. However, the cosmogenic ${ }^{9} \mathrm{Li}$ and ${ }^{8} \mathrm{He}$ background is significant because of the much higher muon rate. The ${ }^{9} \mathrm{Li}$ and 


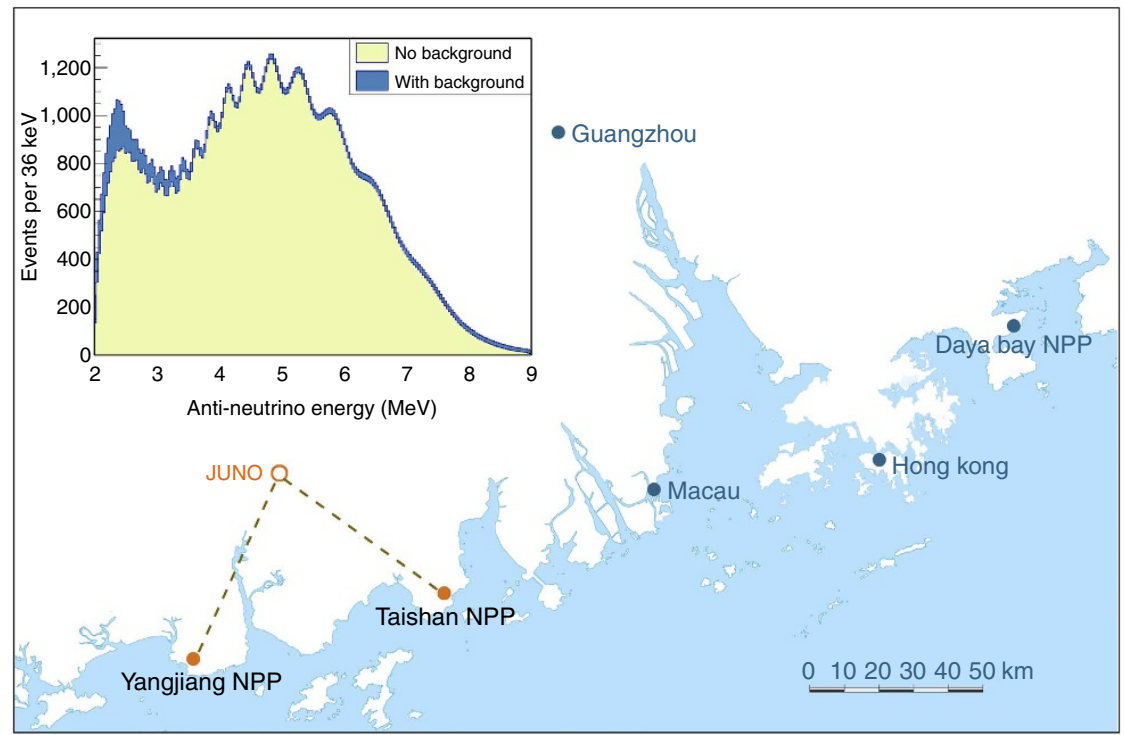

Figure 5 | JUNO's layout and expected signal. JUNO is located in southern China at an equal baseline of $\sim 53 \mathrm{~km}$ from the powerful reactors at Yangjiang and Taishan. The shaded histograms in the inset show the expected $\bar{v}_{e}$ energy spectra at JUNO with and without backgrounds after 6 years' running (calculated based on the information given in ref. 25.) The multiple-oscillation structure allows the determination of neutrino mass hierarchy and precision measurement of the oscillation parameters.

${ }^{8} \mathrm{He}$ isotopes are mostly produced by the muons accompanied by large electromagnetic or hadronic showers ${ }^{68}$. In KamLAND, if a shower muon is tagged, the whole detector is vetoed for $2 \mathrm{~s}$. Such a veto strategy will lead to a significant signal loss at JUNO and RENO-50. Since the lateral distance of the isotopes from the parent muon trajectory is approximately exponential ${ }^{68}$, a small veto region along the muon track can efficiently remove the background with minimal loss of signals. Thus, the ability to track the shower muons is essential, which demands new developments in the muon veto system and improvements on the simulations and reconstructions.

JUNO will observe $\sim 60$ reactor $\bar{v}_{e}$ events per day. The expected energy spectrum is shown in Fig. 5 . The sensitivity of the $\mathrm{MH}$ determination at JUNO is estimated to exceed $3 \sigma$ (for the statistical interpretations see refs 69,70) in 6 years ${ }^{63,71}$. Assuming that the effective mass-squared difference measured by the ongoing accelerator experiments can achieve $1.5-1 \%$ precision $^{72}$, the MH sensitivity at JUNO can be improved to 3.7-4.4 $\sigma$ (ref. 63). RENO-50 has similar sensitivity reaches. In addition to the MH determination, both JUNO and RENO-50 have great potentials in the precision measurements of the neutrino oscillation parameters. The experiments expect to measure $\Delta m_{21}^{2},\left|\Delta m_{31}^{2}\right|$ and $\sin ^{2} \theta_{12}$ to precisions better than $1 \%$. This offers a major step towards the unitarity test of the neutrino mixing matrix ${ }^{73}$ and is important to guide the directions of future experiments and theories.

The next-generation medium-baseline reactor experiments provide a unique opportunity to determine the neutrino $\mathrm{MH}$ with the precision measurement of the reactor neutrino spectrum. Most systematic effects are well understood and studied, although the technical challenges are significant. The MH sensitivity is expected to reach 3-4 $\sigma$. The reactor measurements are independent of $\theta_{23}$, the CP-violating phase and the matter effect. Combining with the future long-baseline accelerator ${ }^{74,75}$ and atmospheric $^{76,77}$ neutrino oscillation programmes, we will once again have complementary measurements of the neutrino $\mathrm{MH}$ with different types of experiments. Such complementarity has proved essential in the history of establishing the phenomenon of neutrino oscillations.

\section{Searching for sterile neutrinos}

Precision electroweak measurements of the decay width of the $Z$ boson determine the number of active light neutrinos. The result, $2.92 \pm 0.05$ (ref. 78), is obviously compatible with the three neutrino flavours. The three-neutrino framework has been extremely successful in explaining neutrino oscillation results, since only two oscillation frequencies, corresponding to the two mass-squared differences $\left(\Delta m_{21}^{2} \sim 7.6 \times 10^{-5} \mathrm{eV}^{2}\right.$ and $\Delta m_{31}^{2} \sim 2.4 \times 10^{-3} \mathrm{eV}^{2}$ ), were observed by the solar, atmospheric, accelerator and reactor neutrino oscillation experiments. However, in the 1990s, the LSND experiment ${ }^{79,80}$ reported an anomalous event excess in the $\bar{v}_{\mu} \rightarrow \bar{v}_{e}$ appearance channel, which could be interpreted as an oscillation with the $\Delta m^{2} \sim 1 \mathrm{eV}^{2}$. Such a scale is clearly incompatible with the above $\Delta m_{21}^{2}$ and $\Delta m_{31}^{2}$. Since the LSND result contradicted the threeneutrino framework, it is often referred to as the 'LSND anomaly'.

The LSND anomaly indicates the existence of additional fourth or more neutrino families with masses $m \sim 1 \mathrm{eV}$. Since these additional neutrinos cannot couple to $Z$ bosons, they must lack weak interactions and are therefore sterile. Sterile neutrinos are observable only through their sub-dominant mixing with the familiar active neutrinos. The light sterile neutrinos, coincidentally, are also among the leading candidates to resolve outstanding puzzles in astrophysics and cosmology ${ }^{81-84}$. On the other hand, the light sterile neutrinos are generally not 'natural' in the theories that extend the neutrino Standard Model. For example, the popular type-I see-saw model ${ }^{85-88}$, which provides an elegant explanation of the small neutrino masses and the matterantimatter asymmetry of the universe ${ }^{89}$, predicts only heavy sterile neutrinos $\left(m>10^{10} \mathrm{eV}\right)$. If the light sterile neutrinos indeed exist, as LSND indicates, they would suggest new frontiers in both experimental and theoretical physics.

The LSND anomaly so-far remains experimentally unconfirmed, despite many efforts. However, there are several hints supporting LSND's findings, even though none are really conclusive. The MiniBooNE experiment, designed at a similar $L / E$ baseline as LSND using accelerator neutrinos, observed event excess in the $v_{\mu} \rightarrow v_{e}$ and $\bar{v}_{\mu} \rightarrow \bar{v}_{e}$ appearance channels that have been interpreted as consistent with LSND ${ }^{90,91}$. The GALLEX $^{92}$ 
Table 2 | Key parameters of the very short-baseline reactor experiments.

\begin{tabular}{|c|c|c|c|c|c|}
\hline & Power $\left(\mathbf{M W}_{\mathrm{th}}\right)$ & Baseline (m) & Mass (ton) & Dopant & Seg. \\
\hline PROSPECT ${ }^{118}$ & 85 & $6-20$ & 1 and 10 & ${ }^{6} \mathrm{Li}$ & $\mathrm{Y}$ \\
\hline NuLat ${ }^{119}$ & 1,500 & $3-8$ & 1.0 & ${ }^{10} \mathrm{~B},{ }^{6} \mathrm{Li}$ & $Y$ \\
\hline NUCIFER ${ }^{120}$ & 70 & $\sim 7$ & 0.7 & Gd & $\mathrm{N}$ \\
\hline STEREO ${ }^{108}$ & 57 & $\sim 10$ & 1.8 & $\mathrm{Gd}$ & $\mathrm{N}$ \\
\hline DANSS $^{122}$ & 3,000 & $9-12$ & 0.9 & $\mathrm{Gd}$ & $Y$ \\
\hline NEUTRINO-4 (ref. 123) & 100 & $6-12$ & 1.5 & $\mathrm{Gd}$ & $\mathrm{N}$ \\
\hline POSEIDON ${ }^{125}$ & 100 & $5-8$ & 1.3 & $\mathrm{Gd}$ & $\mathrm{N}$ \\
\hline SOLID 126 & $45-80$ & 6.8 & 2.9 & $\mathrm{Gd},{ }^{6} \mathrm{Li}$ & Y \\
\hline HANARO $^{127}$ & 30 & 6 & $\sim 1$ & $\mathrm{Gd}$ & $Y$ \\
\hline
\end{tabular}

and the $\mathrm{SAGE}^{93}$ solar neutrino experiments, during their calibrations using intense neutrino sources $\left({ }^{51} \mathrm{Cr},{ }^{37} \mathrm{Ar}\right)$, observed an $\sim 24 \%$ event deficit in the $v_{e}$ disappearance channel. This deficit is often referred to as the 'Gallium anomaly'. Recently, re-evaluations of the reactor $\bar{v}_{e}$ flux resulted in an increase in the predicted $\bar{v}_{e}$ rate ${ }^{37,39}$ (see earlier sections for details). Combining the new predictions with the reactor experimental data at baselines between 10 and $100 \mathrm{~m}$ (refs 9-15) suggests an $\sim 4-6 \%$ deficit between the measured and predicted reactor $\bar{v}_{e}$ flux, so-called 'reactor antineutrino anomaly ${ }^{27,94}$. These experimental anomalies can be interpreted by light-sterile neutrinos ${ }^{95}$, but might also be caused by an imperfect knowledge of the theoretical predictions or experimental systematics. The preferred region $\left(\Delta m^{2} \sim 1 \mathrm{eV}^{2}\right.$ and $\left.\sin ^{2} 2 \theta \sim 0.1\right)$, however, is in some tension with the limits derived from other appearance ${ }^{96-99}$ or disappearance searches ${ }^{15,100-107}$.

There is, therefore, a strong motivation, despite the rather confusing present experimental status, to search for the lightsterile neutrinos. This led to a high-priority world-wide programme $^{108}$ with many proposed next-generation neutrino oscillation experiments. Different technologies will be used, including short-baseline accelerator experiments ${ }^{109-113}$ with various neutrino beams, ${ }^{51} \mathrm{Cr}\left({ }^{144} \mathrm{Ce}-{ }^{144} \mathrm{Pr}\right) v_{e}\left(\bar{v}_{e}\right)$ source experiments $^{114-117}$ near or inside large LS detectors, as well as very short-baseline $(\sim 10 \mathrm{~m})$ reactor (VSBR) $\bar{v}_{e}$ experiments. In order to unambiguously resolve the LSND anomaly, the oscillation pattern in the $L / E$ space need to be observed, as in KamLAND (Fig. 3) and Daya Bay (Fig. 4). VSBR experiments provide unique opportunities to do so, given the many advantages provided by reactors.

Multiple VSBR experiments have been proposed globally; in the United States (PROSPECT ${ }^{118}$, NuLat ${ }^{119}$ ), France (NUCIFER $^{120,121}$, STEREO ${ }^{108}$ ), Russia (DANSS ${ }^{122}$, NEUTRINO-4 (ref. 123,124), POSEIDON ${ }^{125}$ ), United Kingdom $\left(\right.$ SOLID $\left.^{126}\right)$ and Korea (HANARO ${ }^{127}$ ). Table 2 summarizes some of the key parameters of the proposed VSBR experiments. The oscillation length of the $\sim 1-\mathrm{eV}$ mass-scale sterile neutrinos is $\sim 10 \mathrm{~m}$ for reactor $\bar{v}_{e}$ 's; thus, all proposed experiments deploy their detectors at distances of 4-20 m from the reactor cores. The reactor cores should preferably be compact in size to minimize the oscillations inside the cores; therefore, most experiments utilize compact research reactors with thermal power of tens of megawatts. Those research reactors are typically highly enriched in ${ }^{235} \mathrm{U}$, in contrast to the commercial reactors in the NPPs.

Background control is a challenging task in the VSBR experiments. The detectors are typically at shallow depth $(\sim 10$ m.w.e.) constrained by the locations of the reactor cores. The cosmic-ray-related background is therefore high. One advantage of using research reactors is that they can be turned on or off on demand, which helps to measure the nonreactor background. The reactor-related backgrounds, such as fast neutrons and high-energy gamma rays, are however more difficult to determine as they appear together with the $\bar{v}_{e}$ signals. Sufficient active veto and passive shielding are necessary. However, given the tight space near the reactor cores, they have to be carefully designed.

As shown in Table 2, detectors are typically Gd-loaded or ${ }^{6}$ Li-loaded liquid (or solid) scintillators. The Gd-LS technology is mature and a good pulse shape discrimination against the neutron background has been demonstrated. An advantage of the ${ }^{6} \mathrm{Li}$-loaded scintillator is that the delayed neutron capture process ${ }^{6} \mathrm{Li}(n, \alpha) t$ produces an $\alpha$ particle and a triton, instead of a $\gamma$-ray. This provides a good localization of the delayed signal and an additional pulse shape discrimination against the $\gamma$-background. Some detectors are highly segmented into small cells in order to achieve good position resolution and further background rejection by using the multicell event topologies. There are, however, more inactive layers in the segmented detectors; therefore, the edge effects have to be accurately simulated and measured. It is also more challenging to perform calibrations and control the relative variations among cells for the segmented detectors. For all detectors, sufficient light yield is required to precisely measure the reactor $\bar{v}_{e}$ spectrum and the possible distortions from neutrino oscillations.

Despite the challenges, very short-baseline reactor experiments provide a great opportunity to observe the distinctive feature of the light-sterile neutrino oscillations because of their extended range of energy (1-8 MeV) and baselines (5-20 m). The worldwide next-generation VSBR experiments, as shown in Table 2, are being actively considered and pursued. Many of them will begin taking data ${ }^{126}$ in $2015-2016$. Within a few years' running, they expect to cover the parameter region suggested by the experimental anomalies with a sensitivity better than $5 \sigma$ and may tell us whether the fascinating possibility of light-sterile neutrinos is true or not.

\section{Outlook}

Over the past $\sim 60$ years, nuclear reactors have proven to be one of the most powerful tools to study neutrino oscillations, the quantum-mechanical phenomenon that requires extensions to the Standard Model. Experiments at a few kilometres and at a few hundred kilometres from the reactor cores have produced some of the most convincing proofs of neutrino oscillations, by observing the oscillatory behaviour of the reactor $\bar{v}_{e}$ 's in the $L / E$ domain during their propagation. Reactor experiments measured several key parameters governing the neutrino mixing, including $\theta_{12}, \theta_{13}, \Delta m_{21}^{2}$ and $\left|\Delta m_{31}^{2}\right|$. They are essential in establishing the framework of neutrino oscillations. 
Nuclear reactors will continue to help us uncover more facts about neutrinos. In the next $\sim 20$ years, the upcoming nextgeneration reactor experiments will tell us what is the neutrino $\mathrm{MH}$ and whether or not light-sterile neutrinos exist. The results will have significant impact on other future programmes such as neutrinoless double-beta decay experiments, long-baseline accelerator experiments, astrophysics and cosmology. Ultimately, they may hold the key to our deeper understanding of fundamental physics and the universe.

\section{References}

1. Olive, K. A. et al. (Particle Data Group), The Review of Particle Physics. Chin. Phys. C 38, 090001 (2014).

2. Pontecorvo, B. Mesonium and anti-mesonium. Sov. Phys. JETP 6, 429 (1957).

3. Pontecorvo, B. Inverse beta processes and nonconservation of lepton charge. Sov. Phys. JETP 7, 172-173 (1958).

4. Maki, Z., Nakagawa, M. \& Sakata, S. Remarks on the unified model of elementary particles. Prog. Theor. Phys. 28, 870-880 (1962).

5. Pauli, W. Letter to a physicist's gathering at Tübingen, December 4, 1930. Unpublished, the letter was reproduced in L. M. Brown, Physics Today 31, 9 (1978).

The letter that first predicted the existence of neutrinos in order to explain the continuous electron energy distribution in nuclear beta decay.

6. Reines, F. \& Cowan, C. L. Detection of the free neutrino. Phys. Rev. 92, 830-831 (1953).

7. Cowan, C., Reines, F., Harrison, F., Kruse, H. \& McGuire, A. Detection of the free neutrino: a confirmation. Science 124, 103-104 (1956).

8. Reines, F. \& Cowan, C. L. Free antineutrino absorption cross section. I. Measurement of the free antineutrino absorption cross section by protons. Phys. Rev. 113, 273-279 (1959).

Final report on the discovery of neutrinos using the nuclear reactor as their source.

9. Kwon, H. et al. Search for neutrino oscillations at a fission reactor. Phys. Rev. D 24, 1097-1111 (1981).

10. Zacek, G. et al. Neutrino-oscillation experiments at the Gösgen nuclear power reactor. Phys. Rev. D 34, 2621-2636 (1986).

11. Afonin, A. I. et al. A study of the reaction $\bar{v}_{e}+p \rightarrow e^{+}+n$ on a nuclear reactor. J. Exp. Theor. Phys. 67, 213 (1988).

12. Vidyakin, G. S. et al. A study of the reaction $\bar{v}_{e}+p \rightarrow e^{+}+n$ on a nuclear reactor. JETP Lett. 59, 390-393 (1994).

13. Greenwood, Z. D. et al. Results of a two-position reactor neutrino-oscillation experiment. Phys. Rev. D 53, 6054-6064 (1996).

14. Declais, Y. et al. Study of reactor antineutrino interaction with proton at Bugey nuclear power plant. Phys. Lett. B 338, 383-389 (1994).

15. Declais, Y. et al. Search for neutrino oscillations at 15, 40, and 95 meters from a nuclear power reactor at Bugey. Nucl. Phys. B 434, 503-534 (1995).

16. Bemporad, C., Gratta, G. \& Vogel, P. Reactor-based neutrino oscillation experiments. Rev. Mod. Phys. 74, 297-328 (2002).

Review of the early short baseline reactor neutrino experiments.

17. Eguchi, K. et al. First results from KamLAND: evidence for reactor antineutrino disappearance. Phys. Rev. Lett. 90, 021802 (2003).

18. Araki, T. et al. Measurement of neutrino oscillation with KamLAND: evidence of spectral distortion. Phys. Rev. Lett. 94, 081801 (2005).

19. Abe, S. et al. Precision measurement of neutrino oscillation parameters with KamLAND. Phys. Rev. Lett. 100, 221803 (2008).

Observation in the KamLAND experiment of the "solar" neutrino oscillations using the reactors and accurate determination of the $\Delta \mathrm{m}_{21}^{2}$.

20. An, F. P. et al. Observation of electron-antineutrino disappearance at Daya Bay. Phys. Rev. Lett. 108, 171803 (2012).

21. An, F. P. et al. Spectral measurement of electron antineutrino oscillation amplitude and frequency at Daya Bay. Phys. Rev. Lett. 112, 061801 (2014). Determination of the mixing angle $\theta_{13}$ and observation of the associated oscillations at the Daya-Bay reactor experiment.

22. Ahn, J. K. et al. Observation of reactor electron antineutrinos disappearance in the RENO experiment. Phys. Rev. Lett. 108, 191802 (2012).

23. Abe, Y. et al. Indication of reactor $\bar{v}_{e}$ disappearance in the Double Chooz experiment. Phys. Rev. Lett. 108, 131801 (2012).

24. Abe, Y. et al. Background-independent measurement of $\theta_{13}$ in Double Chooz. Phys. Lett. B 735, 51-56 (2014).

25. He, M. Jiangmen Underground Neutrino Observatory. Preprint at http:// arxiv.org/abs/1412.4195 (2014)

26. Kim, S. B. New results from RENO and prospects with RENO-50. Preprint at http://arxiv.org/abs/1412.2199 (2013).

27. Mention, G. et al. Reactor antineutrino anomaly. Phys. Rev. D 83, 073006 (2011).
Reanalysis of the short baseline reactor neutrino experiments; indications of the missing flux interpreted as the possible signal of the sterile neutrinos.

28. von Feilitzsch, F., Hahn, A. \& Schreckenbach, K. Experimental beta-spectra from ${ }^{239} \mathrm{Pu}$ and ${ }^{235} \mathrm{U}$ thermal neutron fission products and their correlated antineutrino spectra. Phys. Lett. B 118, 162-166 (1982).

29. Schreckenbach, K., Colvin, G., Gelletly, W. \& von Feilitzsch, F. Determination of the antineutrino spectrum from ${ }^{235} \mathrm{U}$ thermal neutron fission products up to $9.5 \mathrm{MeV}$. Phys. Lett. B 160, 325-330 (1985).

Measurement of the electron spectra associated with fission and their conversion into the antineutrino spectrum.

30. Hahn, A. et al. Antineutrino spectra from ${ }^{241} \mathrm{Pu}$ and ${ }^{239} \mathrm{Pu}$ thermal neutron fission products. Phys. Lett. B 218, 365-368 (1989).

31. Haag, N. et al. Experimental determination of the antineutrino spectrum of the fission products of ${ }^{238} \mathrm{U}$. Phys. Rev. Lett. 112, 122501 (2014).

32. Davis, B. R., Vogel, P., Mann, F. M. \& Schenter, R. E. Reactor antineutrino spectra and their application to antineutrino-induced reactions. Phys. Rev. C 19, 2259-2266 (1979)

33. Vogel, P., Schenter, G. K., Mann, F. M. \& Schenter, R. E. Reactor antineutrino spectra and their application to antineutrino-induced reactions. II. Phys. Rev. C 24, 1543-1553 (1981).

34. Klapdor, H. V. \& Metzinger, J. Antineutrino spectrum from the fission products of ${ }^{239} \mathrm{Pu}$. Phys. Rev. Lett. 48, 127-131 (1982).

35. Klapdor, H. \& Metzinger, J. Calculation of the antineutrino spectrum from thermal fission of ${ }^{235} \mathrm{U}$. Phys. Lett. B 112, 22-26 (1982).

36. Kopeikin, V. I. Electron and antineutrino spectra from fragments of fission of ${ }^{239} \mathrm{U},{ }^{239} \mathrm{Pu},{ }^{241} \mathrm{Pu}$ induced by thermal neutrons and of fission of ${ }^{238} \mathrm{U}$ induced by fast neutrons. Sov. J. Nucl. Phys. 32, 780 (1980).

37. Mueller, T. A. et al. Improved predictions of reactor antineutrino spectra. Phys. Rev. C 83, 054615 (2011).

Reevaluation of the reactor antineutrino spectrum resulting in $\sim 3 \%$ increase of the flux.

38. Vogel, P. Conversion of electron spectrum associated with fission into the antineutrino spectrum. Phys. Rev. C 76, 025504 (2007).

39. Huber, P. Determination of antineutrino spectra from nuclear reactors. Phys. Rev. C 84, 024617 (2011).

40. Hayes, A. C., Friar, J. L., Garvey, G. T., Jungman, G. \& Jonkmans, G. Systematic uncertainties in the analysis of the reactor neutrino anomaly. Phys. Rev. Lett. 112, 202501 (2014).

Inclusion of the first forbidden beta decays of the fission fragments increases the uncertainty of the reactor spectrum normalization to $\sim 5 \%$.

41. Vogel, P. \& Beacom, J. F. Angular distribution of neutron inverse beta decay, $\bar{v}_{e}+p \rightarrow e^{+}+n$. Phys. Rev. D 60, 053003 (1999).

42. Strumia, A. \& Vissani, F. Precise quasielastic neutrino/nucleon cross-section. Phys. Lett. B 564, 42-54 (2003).

43. Davis, R., Harmer, D. S. \& Hoffman, K. C. Search for neutrinos from the Sun. Phys. Rev. Lett. 20, 1205-1209 (1968).

First experiment that measured the solar neutrinos. The deficit in the measured flux with respect to standard solar model prediction indicated the "solar neutrino problem".

44. Hampel, W. et al. GALLEX solar neutrino observations: results for GALLEX IV. Phys. Lett. B 447, 127-133 (1999).

45. Abdurashitov, J. N. et al. Measurement of the solar neutrino capture rate by SAGE and implications for neutrino oscillations in vacuum. Phys. Rev. Lett. 83, 4686-4689 (1999).

46. Hirata, K. S. et al. Observation of ${ }^{8} \mathrm{~B}$ solar neutrinos in the Kamiokande-II detector. Phys. Rev. Lett. 63, 16-19 (1989).

47. Fukuda, Y. et al. Measurements of the solar neutrino flux from SuperKamiokande's first 300 days. Phys. Rev. Lett. 81, 1158-1162 (1998).

48. Bahcall, J. N. Solar neutrino cross sections and nuclear beta decay. Phys. Rev. 135, B137-B146 (1964).

49. Ahmad, Q. R. et al. Direct evidence for neutrino flavor transformation from neutral-current interactions in the Sudbury Neutrino Observatory. Phys. Rev. Lett. 89, 011301 (2002).

Experimental proof of the flavour change of the solar neutrinos by determination of the total flux of solar neutrinos using the neutral current reaction.

50. Berger, B. E. et al. The KamLAND full-volume calibration system. J. Instrum. 4, P04017 (2009)

51. Kajita, T. \& Totsuka, Y. Observation of atmospheric neutrinos. Rev. Mod. Phys. 73, 85 (2001).

52. Feldman, G., Hartnell, J. \& Kobayashi, T. Long-baseline neutrino oscillation experiments. Adv. High Energy Phys. 2013, 475749 (2013).

53. Apollonio, M. et al. Limits on neutrino oscillations from the $\mathrm{CHOOZ}$ experiment. Phys. Lett. B 466, 415-430 (1999).

54. Boehm, F. et al. Search for neutrino oscillations at the Palo Verde nuclear reactors. Phys. Rev. Lett. 84, 3764-3767 (2000).

55. Harrison, P. F., Perkins, D. H. \& Scott, W. G. Tri-bimaximal mixing and the neutrino oscillation data. Phys. Lett. B 530, 167-173 (2002). 
56. Altarelli, G. \& Feruglio, F. Discrete flavor symmetries and models of neutrino mixing. Rev. Mod. Phys. 82, 2701-2729 (2010).

57. Zhang, C. Recent results from the Daya Bay experiment. Preprint at http:// arxiv.org/abs/1501.04991 (2015).

58. Adamson, P. et al. Combined analysis of $v_{\mu}$ disappearance and $v_{\mu} \rightarrow v_{e}$ appearance in MINOS using accelerator and atmospheric neutrinos. Phys. Rev. Lett. 112, 191801 (2014).

59. Abe, K. et al. Precise measurement of the neutrino mixing parameter $\theta_{23}$ from muon neutrino disappearance in an off-axis beam. Phys. Rev. Lett. 112, 181801 (2014).

60. Abe, K. et al. Indication of electron neutrino appearance from an acceleratorproduced off-axis muon neutrino beam. Phys. Rev. Lett. 107, 041801 (2011).

61. Adamson, P. et al. Improved search for muon-neutrino to electron-neutrino oscillations in MINOS. Phys. Rev. Lett. 107, 181802 (2011).

62. Cahn, R. N. et al. White Paper: measuring the neutrino mass hierarchy. Preprint at http://arxiv.org/abs/1307.5487 (2013).

63. Li, Y., Cao, J., Wang, Y. \& Zhan, L. Unambiguous determination of the neutrino mass hierarchy using reactor neutrinos. Phys. Rev. D 88, 013008 (2013).

64. Zhan, L., Wang, Y., Cao, J. \& Wen, L. Determination of the neutrino mass hierarchy at an intermediate baseline. Phys. Rev. D 78, 111103 (2008).

65. Zhan, L., Wang, Y., Cao, J. \& Wen, L. Experimental requirements to determine the neutrino mass hierarchy using reactor neutrinos. Phys. Rev. D 79, 073007 (2009).

66. Minakata, H., Nunokawa, H., Parke, S. J. \& Funchal, R. Z. Determining neutrino mass hierarchy by precision measurements in electron and muon neutrino disappearance experiments. Phys. Rev. D 74, 053008 (2006).

67. Qian, X. et al. Mass hierarchy resolution in reactor anti-neutrino experiments: parameter degeneracies and detector energy response. Phys. Rev. D 87, 033005 (2013).

68. Abe, S. et al. Production of radioactive isotopes through cosmic muon spallation in KamLAND. Phys. Rev. C 81, 025807 (2010).

69. Qian, X. et al. Statistical evaluation of experimental determinations of neutrino mass hierarchy. Phys. Rev. D 86, 113011 (2012).

70. Blennow, M., Coloma, P., Huber, P. \& Schwetz, T. Quantifying the sensitivity of oscillation experiments to the neutrino mass ordering. J. High Energy Phys. 2014, 028 (2014)

71. Kettell, S. et al. Neutrino mass hierarchy determination and other physics potential of medium-baseline reactor neutrino oscillation experiments. Preprint at http://arxiv.org/abs/1307.7419 (2013).

72. Agarwalla, S. K., Prakash, S. \& Wang, W. High-precision measurement of atmospheric mass-squared splitting with T2K and NOvA. Preprint at http:// arxiv.org/abs/1312.1477 (2013).

73. Qian, X., Zhang, C., Diwan, M. \& Vogel, P. Unitarity tests of the neutrino mixing matrix. Preprint at http://arxiv.org/abs/1308.5700 (2013).

74. Adams, C. et al. The long-baseline neutrino experiment: exploring fundamental symmetries of the universe. Preprint at http://arxiv.org/abs/ 1307.7335 (2013)

75. Agarwalla, S. et al. The mass-hierarchy and CP-violation discovery reach of the LBNO long-baseline neutrino experiment. J. High Energy Phys. 1405, 094 (2014).

76. Aartsen, M. et al. Letter of intent: the precision IceCube next generation upgrade (PINGU). Preprint at http://arxiv.org/abs/1401.2046 (2014).

77. Katz, U. F. et al. The ORCA option for KM3NeT. Preprint at http://arxiv.org/ abs/1402.1022 (2014).

78. Schael, S. et al. (ALEPH Collaboration, DELPHI Collaboration, L3 Collaboration, OPAL Collaboration, SLD Collaboration, LEP Electroweak Working Group, SLD Electroweak Group, SLD Heavy Flavour Group), Precision Electroweak Measurements on the Z Resonance. Phys. Rep. 427, 257-454 (2006).

79. Athanassopoulos, C. et al. Candidate events in a search for $\bar{v}_{\mu} \rightarrow \bar{v}_{e}$ oscillations. Phys. Rev. Lett. 75, 2650-2653 (1995).

80. Aguilar, A. et al. Evidence for neutrino oscillations from the observation of $\bar{v}_{e}$ appearance in a $\bar{v}_{\mu}$ beam. Phys. Rev. D 64, 112007 (2001).

Indication of the existence of light sterile neutrinos in the LSND experiment.

81. Dodelson, S. \& Widrow, L. M. Sterile neutrinos as dark matter. Phys. Rev. Lett. 72, 17-20 (1994)

82. Kusenko, A. Sterile neutrinos: The Dark side of the light fermions. Phys. Rep. 481, 1-28 (2009)

83. Wyman, M., Rudd, D. H., Vanderveld, R. A. \& Hu, W. Neutrinos help reconcile planck measurements with the local universe. Phys. Rev. Lett. 112, 051302 (2014)

84. Battye, R. A. \& Moss, A. Evidence for massive neutrinos from cosmic microwave background and lensing observations. Phys. Rev. Lett. 112, 051303 (2014).

85. Minkowski, P. $\mu \rightarrow e \gamma$ at a rate of one out of $10^{9}$ muon decays? Phys. Lett. B 67, 421-428 (1977).
86. Yanagida, T. Horizontal symmetry and masses of neutrinos. Conf. Proc. C7902131, 95-99 (1979).

87. Gell-Mann, M., Ramond, P. \& Slansky, R. Complex spinors and unified theories. Conf. Proc. C790927, 315-321 (1979).

88. Mohapatra, R. N. \& Senjanović, G. Neutrino mass and spontaneous parity nonconservation. Phys. Rev. Lett. 44, 912-915 (1980).

89. Fukugita, M. \& Yanagida, T. Barygenesis without grand unification. Phys. Lett. B 174, 45-47 (1986).

90. Aguilar-Arevalo, A. A. et al. Search for electron neutrino appearance at the $\Delta m^{2} \sim 1 \mathrm{eV}^{2}$ scale. Phys. Rev. Lett. 98, 231801 (2007).

91. Aguilar-Arevalo, A. A. et al. Improved search for $\bar{v}_{\mu} \rightarrow \bar{v}_{e}$ oscillations in the MiniBooNE experiment. Phys. Rev. Lett. 110, 161801 (2013).

92. Kaether, F. et al. Reanalysis of the GALLEX solar neutrino flux and source experiments. Phys. Lett. B 685, 47-54 (2010).

93. Abdurashitov, J. N. et al. Measurement of the solar neutrino capture rate with gallium metal. III. Results for the 2002-2007 data-taking period. Phys. Rev. C 80, 015807 (2009)

94. Zhang, C., Qian, X. \& Vogel, P. Reactor antineutrino anomaly with known $\theta_{13}$. Phys. Rev. D 87, 073018 (2013).

95. Giunti, C. \& Laveder, M. Status of $3+1$ neutrino mixing. Phys. Rev. D 84, 093006 (2011).

96. Armbruster, B. et al. Upper limits for neutrino oscillations $\bar{v}_{\mu} \rightarrow \bar{v}_{e}$ from muon decay at rest. Phys. Rev. D 65, 112001 (2002).

97. Astier, P. et al. Search for $v_{\mu} \rightarrow v_{e}$ oscillations in the NOMAD experiment. Phys. Lett. B 570, 19-31 (2003).

98. Agafonova, N. et al. Addendum: search for $v_{\mu} \rightarrow v_{e}$ oscillations with the OPERA experiment in the CNGS beam. J. High Energy Phys. 07, 085 (2013)

99. Antonello, M. et al. Search for anomalies in the $v_{e}$ appearance from a $v_{\mu}$ beam. Eur. Phys. J. C 73, 2599 (2013).

100. Stockdale, I. E. et al. Limits on muon-neutrino oscillations in the mass range $30<\Delta m^{2}<1000 \mathrm{eV}^{2} / c^{4}$. Phys. Rev. Lett. 52, 1384-1388 (1984).

101. Dydak, F. et al. A search for $v_{\mu}$ oscillations in the $\Delta m^{2}$ range $0.3-90 \mathrm{eV}^{2}$. Phys. Lett. B 134, 281-286 (1984).

102. Cheng, G. et al. Dual baseline search for muon antineutrino disappearance at $0.1 \mathrm{eV}^{2}<\Delta m^{2}<100 \mathrm{eV}^{2}$. Phys. Rev. D 86, 052009 (2012).

103. Mahn, K. B. M. et al. Dual baseline search for muon neutrino disappearance at $0.5 \mathrm{eV}^{2}<\Delta m^{2}<40 \mathrm{eV}^{2}$. Phys. Rev. D 85, 032007 (2012).

104. Fukuda, S. et al. Tau neutrinos favored over sterile neutrinos in atmospheric muon neutrino oscillations. Phys. Rev. Lett. 85, 3999-4003 (2000).

105. Adamson, P. et al. Active to sterile neutrino mixing limits from neutralcurrent interactions in MINOS. Phys. Rev. Lett. 107, 011802 (2011).

106. Conrad, J. M. \& Shaevitz, M. H. Limits on electron neutrino disappearance from the KARMEN and LSND $v_{e}$-carbon cross section data. Phys. Rev. D 85, 013017 (2012).

107. An, F. P. et al. Search for a light sterile neutrino at Daya Bay. Phys. Rev. Lett. 113, 141802 (2014).

108. Abazajian, K. N. et al. Light sterile neutrinos: a white paper. Preprint at http:// arxiv.org/abs/1204.5379 (2012).

109. Bungau, A. et al. Proposal for an electron antineutrino disappearance search using high-rate ${ }^{8}$ Li production and decay. Phys. Rev. Lett. 109, 141802 (2012).

110. Allen, R. et al. The OscSNS White Paper. Preprint at http://arxiv.org/abs/ 1307.7097 (2013).

111. Antonello, M. et al. Search for 'anomalies' from neutrino and anti-neutrino oscillations at $\Delta m^{2} \sim 1 \mathrm{eV}^{2}$ with muon spectrometers and large LAr-TPC imaging detectors. Preprint at http://arXiv.org/abs/1203.3432 (2012).

112. Adams, C. et al. LAr1-ND: testing neutrino anomalies with multiple LArTPC detectors at Fermilab. Preprint at http://arXiv/abs/1309.7987 (2013).

113. Adey, D. et al. Light sterile neutrino sensitivity at the nuSTORM facility. Phys. Rev. D 89, 071301 (2014).

114. Cribier, M. et al. Proposed search for a fourth neutrino with a PBq antineutrino source. Phys. Rev. Lett. 107, 201801 (2011).

115. Dwyer, D. A., Heeger, K. M., Littlejohn, B. R. \& Vogel, P. Search for sterile neutrinos with a radioactive source at Daya Bay. Phys. Rev. D 87, 093002 (2013).

116. Bellini, G. et al. SOX: Short distance neutrino Oscillations with BoreXino. J. High Energy Phys. 08, 038 (2013).

117. Gando, A. et al. CeLAND: search for a 4th light neutrino state with a $3 \mathrm{PBC}$ ${ }^{144} \mathrm{Ce}^{144} \mathrm{Pr}$ electron antineutrino generator in KamLAND. Preprint at http:// arxiv.org/abs/1312.0896 (2013).

118. Ashenfelter, J. et al. PROSPECT-a precision reactor oscillation and spectrum experiment at very short baselines. Preprint at http://arxiv.org/abs/1309.7647 (2013).

119. Lane, C. et al. A new type of neutrino detector for sterile neutrino search at nuclear reactors and nuclear nonproliferation applications. Preprint at http:// arxiv.org/abs/1501.06935 (2015).

120. Porta, A. et al. Reactor neutrino detection for non-proliferation with the NUCIFER experiment. IEEE Trans. Nucl. Sci. 57, 2732 (2010). 
121. Cucoanes, A. S. et al. Status of the Nucifer experiment. J. Phys. Conf. Ser. 375, 042063 (2012).

122. Alekseev, I. et al. DANSSino: a pilot version of the DANSS neutrino detector. Phys. Part. Nucl. Lett. 11, 473-482 (2014).

123. Serebrov, A. P. et al. 'Neutrino-4' experiment: preparations for search for sterile neutrino at $100 \mathrm{MW}$ reactor SM-3 at 6-13 meters. Preprint at http:// arxiv.org/abs/1205.2955 (2012).

124. Serebrov, A. P. et al. On possibility of realization NEUTRINO-4 experiment on search for oscillations of the reactor antineutrino into a sterile state. Preprint at http://arxiv.org/abs/1310.5521 (2014).

125. Derbin, A. V., Kayunov, A. S. \& Muratova, V. N. Search for neutrino oscillations at a research reactor. Preprint at http://arxiv.org/abs/1204.2449 (2012).

126. Lasserre, T. Light sterile neutrinos in particle physics: experimental status. Phys. Dark Univ. 4, 81-85 (2014).

127. Yeo, S. Development of a gadolinium-loaded liquid scintillator for the Hanaro short baseline prototype detector. J. Korean Phys. Soc. 64, 377-381 (2014).

128. Wolfenstein, L. Neutrino oscillations in matter. Phys. Rev. D 17, 2369-2374 (1978).

129. Mikheev, S. P. \& Smirnov, A. Y. Resonance amplification of oscillations in matter and spectroscopy of solar neutrinos. Sov. J. Nucl. Phys. 42, 913-917 (1985).

\section{Acknowledgements}

We thank X. Qian, D. Jaffe, M. Diwan and S. Kettell for reading the manuscript. The work of C.Z. was supported in part by the Department of Energy under contracts DE-SC0012704. The work of P.V. was supported in part by the National Science Foundation NSF-1205977 and by the Physics Department, California Institute of Technology. The work of L.J.W. was supported in part by the National Natural Science Foundation of China (11205183).

\section{Additional information}

Competing financial interests: The authors declare no competing financial interests.

Reprints and permission information is available online at http://npg.nature.com/ reprintsandpermissions/

How to cite this article: Vogel, P. et al. Neutrino oscillation studies with reactors. Nat. Commun. 6:6935 doi: 10.1038/ncomms7935 (2015).

\section{(c) (i)}

This work is licensed under a Creative Commons Attribution 4.0 International License. The images or other third party material in this article are included in the article's Creative Commons license, unless indicated otherwise in the credit line; if the material is not included under the Creative Commons license, users will need to obtain permission from the license holder to reproduce the material. To view a copy of this license, visit http://creativecommons.org/licenses/by/4.0/ 